\title{
Modified versus conventional Tenzel semicircular flap in congenital bilateral upper eyelid coloboma occurring as an isolated finding: a case series
}

\author{
Shanti Fitrianti Boesoirie, $\mathrm{MD}, \mathrm{PhD}^{1}$ (iD, $\mathrm{M}$ Rinaldi Dahlan, $\mathrm{MD}, \mathrm{PhD}^{1}{ }^{1}$, Angga Kartiwa, $\mathrm{MD}, \mathrm{PhD}^{1}$ (iD, \\ Niluh Putu Ayu, MD'1iD, Woong Chul Choi, MD² \\ ${ }^{1}$ Department of Ophthalmology, Cicendo Eye Hospital, Padjadjaran University, Bandung, Indonesia, ${ }^{2}$ Myoung Eye Clinic, Seoul, Rep. of Korea
}

Congenital eyelid coloboma is a full- or partial-thickness defect of the eyelids caused by failure of fusion of the embryonic fissure during embryological development. The aim of this study is to provide information regarding the modified reverse Tenzel semicircular flap procedure in the management of bilateral upper eyelid coloboma. The conventional Tenzel semicircular flap stabilizes the eyelid margins and ensures adequate eye opening and closing functions. However, a modified Tenzel semicircular flap provides enhanced cosmetic results.

Keywords: eyelid coloboma; Tenzel semicircular flap

\section{Introduction}

Congenital coloboma of the eyelid is a rare disorder with an incidence of 1:10,000 live births. The word coloboma is derived from the Greek word “koloboma”, which means mutilated, curtailed, hole, or defect [1]. The condition is associated with full/ partial-thickness defects of the eyelids, due to failure of fusion of the mesodermal folds during embryological development. Colobomas can occur unilaterally or bilaterally, commonly in the upper eyelid, and can appear as an isolated disorder or in conjunction with other ocular or systemic anomalies [1,2]. Keratopathy due to poor Bell's phenomenon and exposure of the cornea are often identified; therefore, the main aims of management of eyelid colobomas are reapir of the anatomical structure and improvement of the function of the eyelid to protect the eyeball $[3,4]$.
The Tenzel semicircular flap is one of the techniques used to close medium-sized defects of the eyelid, which was introduced in a lower lid defect. Therefore, in this study, we would like to use the term reverse Tenzel semicircular flap. The purpose of this case report was to describe and compare the results of a congenital superior eyelid coloboma after reconstruction using the modified reverse Tenzel semicircular flap technique $[3,5,6]$.

\section{Case report}

\section{Case 1}

A 5-month-old girl was brought to Reconstruction Oculoplasty Oncology Unit, Cicendo Eye Hospital with defects in both of her upper eyelids since birth. Her eyelids could not close completely, there were no complaints of red eye or discharge. Ophthalmological examination showed that the patient could

Received March 31, 2020; Revised May 26, 2020; Accepted September 14, 2020

Corresponding author: Shanti Fitrianti Boesoirie

E-mail: boesoirie.sf@gmail.com

This is an Open Access article distributed under the terms of the Creative Commons Attribution Non-Commercial License (http://creativecommons.org/licenses/by-nc/4.0), which permits unrestricted non-commercial use, distribution, and reproduction in any medium, provided the original work is properly cited.

Copyright ( 2020 Korean Society of Korean Cosmetic Surgery and Medicine (KSKCS \& KCCS). 
fix her gaze on and follow the object effectively, thus demonstrating good eye movement. There was no history of ilness or consumption of certain drugs by the mother during pregnancy.

Anterior segment examination revealed a $9 \mathrm{~mm}$ full-thickness defect involving one-third of the upper eyelid bilaterally (Fig. 1). There was no sign of exposure keratitis, iris coloboma, or choroidal coloboma, and there were no anomalies in other organs. The patient was diagnosed as a case of isolated congenital bilateral upper eyelid coloboma, for which surgical repair was planned.

The surgery was performed by the surgeon SFB. A semicircular incision was made starting from the lateral canthus (Fig. 2) using surgical blade no. 15, followed by lateral canthotomy and cantholysis. A temporal myocutaneous flap was formed and pulled medially to approximate the eyelid defect. The modification was then performed by making an additional incision approximately $4 \mathrm{~mm}$ from the lid margin (Fig. 3). Suturing was performed using 6-0 Vicryl (Ethicon, Inc., Somerville, NJ, USA). Postoperatively, the patient was administered an oral analgesic

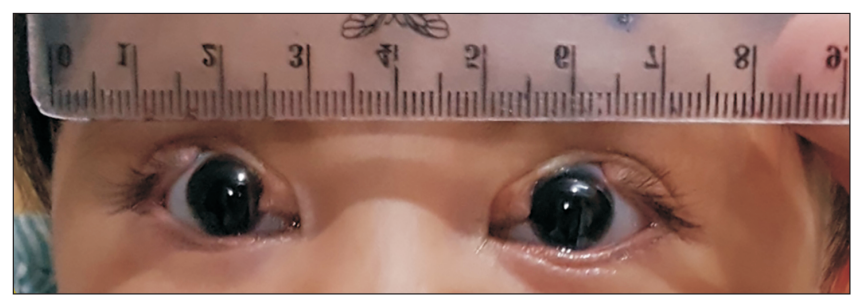

Fig. 1. Clinical appearance of the colobomas before surgery. as well as an oral and topical antibiotic. Postoperative result after 3 months can be seen in Fig. 4.

\section{Case 2}

The second patient was a 2-month-old boy who was brought to Reconstruction Oculoplasty Oncology Unit, Cicendo Eye Hospital with the same complaints as the first patient. Ophthalmological examination revealed a positive blink reflex and
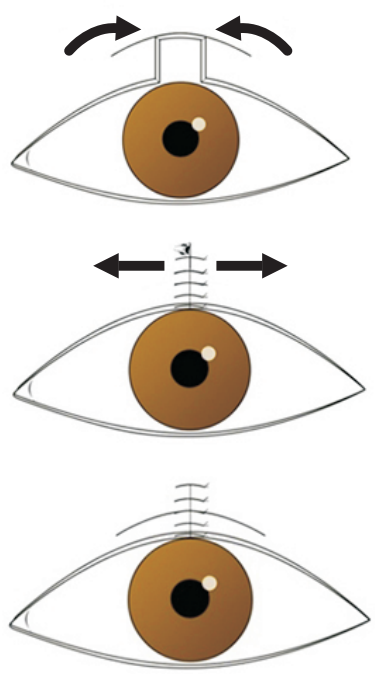

Fig. 3. Schematic eye design shows that after forming and pulling the myocutaneous flap medially and suturing the gap, horizontal tension may occur in the wound with possible dog ear formation. Additional horizontal incision helps to reduce the tension and manage the dog ear.
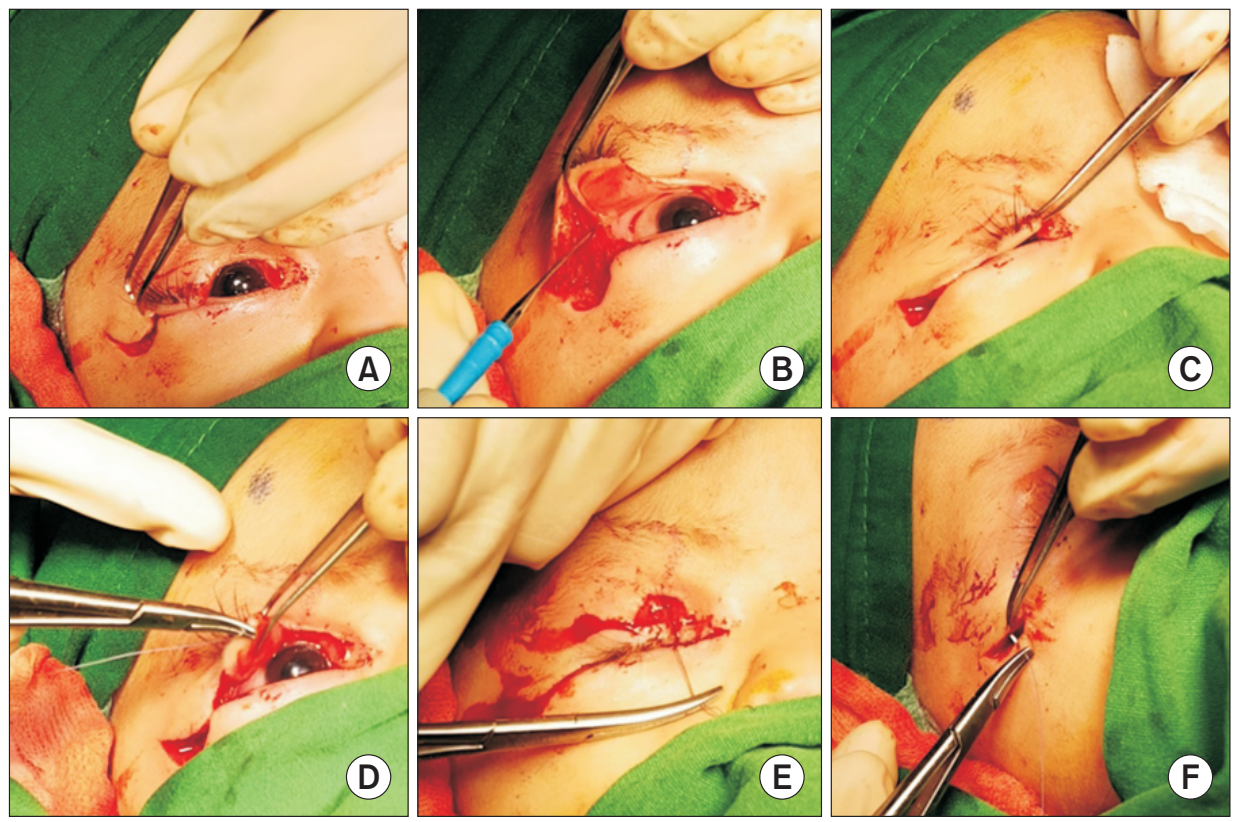

Fig. 2. Surgical steps. (A) Semicircular incision, (B) lateral canthotomy, cantholysis, and formation of a myocutaneous flap, (C) flap pulled medially, (D) suturing the eyelid margins, (E) additional horizontal incision approximately $4 \mathrm{~mm}$ from the lid margin, (F) wound closure. 
a full-thickness defect involving one-third of the upper eyelid, without other colobomas. Mild symblepharon was found in the left eye (Fig. 5).

The surgery was performed by the surgeon MRD using a conventional reverse Tenzel semicircular flap without additional horizontal incision (Fig. 6).

One week postoperatively, wound dehiscence was observed
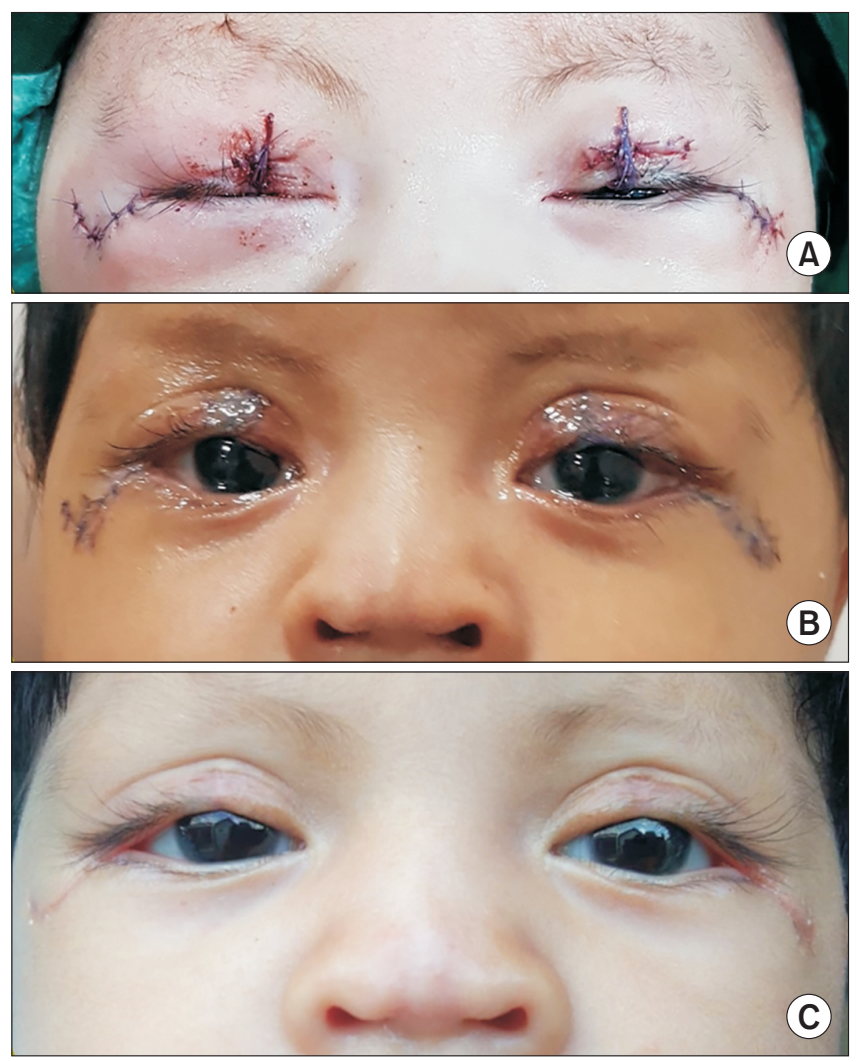

Fig. 4. Clinical appearance on postoperative days 1 (A), days 7 (B), and at 3 months (C). in the right eye that needed re-suturing. The sutures were retained for two weeks before removal.

Informed consent was obtained from the legal guardian of the patient for publication of this case series.

\section{Discussion}

Coloboma of the upper eyelid may pose a threat to vision at a very early stage. The classical congenital eyelid defect usually involves a shortage of the conjunctiva, tarsal plate, orbicularis muscle, and skin that leaves the cornea open and exposed, which leads to exposure keratitis. The goals of treatment are to protect the cornea, improve the structure and function of the eyelids, and visual development. In this manner, upper eyelid coloboma may represent on of the emergency oculoplastic conditions necessitating intervention at an early age, which could be challenging $[2,4,7,8]$.

Management of coloboma depends on the degree of corneal exposure, and defects greater than $35 \%$ may be treated in two stages or by other techniques. Most surgeons prefer the direct closure, Cutler-Beard, canthotomy-cantholysis, or Tenzel flap procedure for coloboma repair $[4,7,9]$.

Both patients in this case series had medium-sized (33\%-50\%

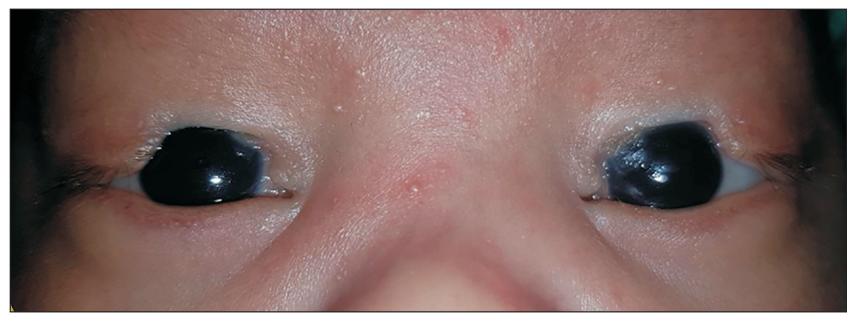

Fig. 5. Coloboma in the second patient.
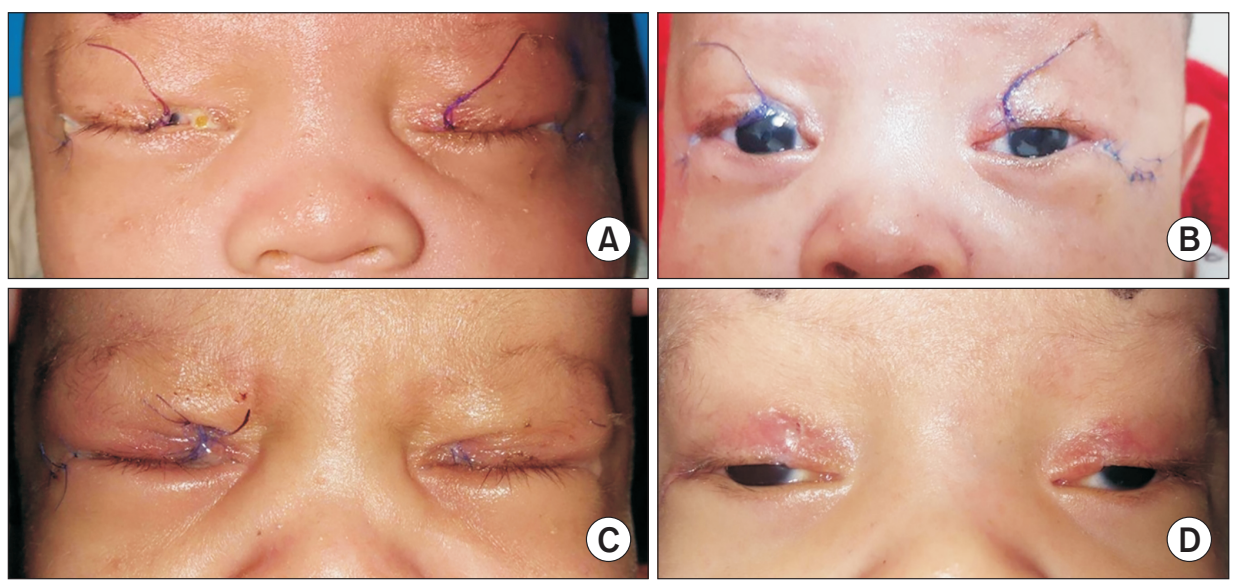

Fig. 6. (A) Clinical appearance one day after surgery, (B) wound dehiscence observed on the 7th postoperative day, (C) after re-suturing, (D) 3 months postoperatively. 
of the length of the eyelid), full-thickness defects on the upper eyelid. We reconstructed the defects using the reverse Tenzel semicircular flap technique. The other reason for choice of the procedure was that both patients were very young; therefore, single step surgery would not have affected their visual axis or posed any risk of amblyopia $[6,10]$. Eyelid reconstruction in infants is a sensitive procedure, as their eyelids are usually very tight. Therefore, lateral canthotomy and superior cantholysis are needed. A $4 \mathrm{~mm}$ horizontal incision was made from the lid margin considering the age of the first patient and the fact that her natural lid crease was high (approximately $10 \mathrm{~mm}$ from the margin). In cases of good height of the natural lid crease, the horizontal incision should be placed at the crease. This horizontal incision has several advantages, such as the ability to form a lid crease in a patient with no crease and reducing the horizontal tension that could lead to wound dehiscence. As seen in the second case, horizontal incision can also reduce narrowing of the eyelid aperture. In addition, this horizontal incision can also relax the skin tension line and facilitate dog ear management. In the first patient, considering the high vertical defect, dog ear repair extended to a superior position, which resulted in change in brow position.

To the best of our knowledge, no study has been conducted on this additional incision in the reverse Tenzel semicircular procedure. This one-step incision is a minor modification, but significantly improves the appearance of the patient after completion of the procedure.

\section{Conclusion}

Bilateral congenital eyelid coloboma is a rare condition that can affect the protective function of the cornea and increase the risk of exposure keratitis. The choice of surgical technique to repair palpebral defects is selected based on the location and vertical and horizontal size of the defect. An additional horizontal incision can be considered in the Tenzel semicircular flap technique to manage coloboma of the upper lid to achieve good visual outcomes and enhanced cosmetic appearance.

\section{Conflicts of interest}

The authors have nothing to disclose.

\section{References}

1. Pagon RA. Ocular coloboma. Surv Ophthalmol 1981;25:22336.

2. Smith HB, Verity DH, Collin JR. The incidence, embryology, and oculofacial abnormalities associated with eyelid colobomas. Eye (Lond) 2015;29:492-8.

3. Khalid M, Moin M, Latif MA. Reconstruction of congenital lid defects. Pak J Ophthalmol 2013;29:231-4.

4. Lodhi AA, Junejo SA, Khanzada MA, Sahaf IA, Siddique ZK. Surgical outcome of 21 patients with congenital upper eyelid coloboma. Int J Ophthalmol 2010;3:69-72.

5. Collin JRO. A manual of systematic eyelid surgery. 3rd ed. Oxford: Elsevier Butterworth-Heinemann; 2006. p. 123.

6. Tenzel RR, Stewart WB. Eyelid reconstruction by the semicircle flap technique. Ophthalmology 1978;85:1164-9.

7. Seah LL, Choo CT, Fong KS. Congenital upper lid colobomas: management and visual outcome. Ophthalmic Plast Reconstr Surg 2002;18:190-5.

8. Tawfik HA, Abdulhafez MH, Fouad YA. Congenital upper eyelid coloboma: embryologic, nomenclatorial, nosologic, etiologic, pathogenetic, epidemiologic, clinical, and management perspectives. Ophthalmic Plast Reconstr Surg 2015;31:112.

9. Ankola PA, Abdel-Azim H. Congenital bilateral upper eyelid coloboma. J Perinatol 2003;23:166-7.

10. [Upper eyelid coloboma treatment with the Cutler-Beard technique and its association with amblyopia: case report]. Arq Bras Oftalmol 2002;65:579-84. Portuguese. 